\title{
Efficiency of Selected Mutagens in Generating Xanthophyllomyces dendrorhous Strains Hyperproducing Astaxanthin
}

\author{
BARBARA STACHOWIAK* \\ Institute of Technology of Plant Origin Food, Poznan University of Life Sciences, Poland
}

Received 22 May 2012, revised 15 November 2012, accepted 15 November 2012

Abstract

Astaxanthin is a xanthophill pigment with commercial application in the aquaculture, pharmaceutical, food and cosmetic industries. The red yeast Xanthophyllomyces dendrorhous is one of the most promising microorganisms for its industrial production. However, astaxanthin content in wild yeast strains is low. Pigment production by X. dendrorhous can be improved by mutagenesis. The aim of the study was to assess the efficiency of four mutagens: UV radiation, benomyl, ethyl methanesulfonate and ethidium bromide in generating asthaxanthinhyperproducing strains of the yeast Xanthophyllomyces dendrorhous DSM 5626. Mutations with benomyl, ethidium bromide and UV radiation generated a group of hyperpigmented mutants exhibiting increases up to $100 \%$ in astaxanthin content. Ethyl methanesulfonate turned out to be useless in this respect.

Key words: Xanthophyllomyces dendrorhous, astaxanthin, carotenoids, mutagenesis

\section{Introduction}

From the moment of its isolation to the present the yeast Xanthophyllomyces dendrorhous (perfect state of Phaffia rhodozyma; Golubev 1995) has been a promising research object. This is connected first of all with its unique ability to synthesize astaxanthin, a pigment from the group of carotenoids. It is extensively used in aquaculture as an integral component of salmon feed (Breithaupt, 2007) and increasingly also in the pharmaceutical, cosmetic and food sector because of its high antioxidant potential and health-promoting properties (Goswami et al., 2010; Rodríguez-Sáiz and de la Fuente, 2010; Yuan et al., 2011).

However, to date the use of $X$. dendrorhous in the commercial scale production of astaxanthin has not been profitable. The pigment content in wild strains of $X$. dendrorhous is low, as it amounts to $0.1-0.25 \mathrm{~g} / \mathrm{kg}$ in dry matter (Flenø et al., 1997). It is solely for this reason that they are not practical or economically feasible sources. A major pre-condition for the application of this yeast in the commercial scale production of astaxanthin is to generate strains with a higher pigment concentration.

Production of astaxanthin by $X$. dendrorhous is most frequently improved by mutagenesis. However, mutations may lead to the generation of strains incapable of synthesis of carotenoids, the so-called albino strains accumulating colourless phytoene, which is a precursor for the synthesis of carotenoids. In this case they relate to genes coding phytoene dehydrogenase/desaturase. Cases have also been reported when strains producing solely $\beta$-carotene were obtained, when mutation occurred at the stage of $\beta$-carotene oxidation to astaxanthin (Girard et al., 1997).

Many chemical or non-chemical mutagens may induce genetic changes in the yeast $X$. dendrorhous. They may be applied independently or in combination. In research nitrosoguanidine is the most frequently applied mutagen of $X$. dendrorhous (Ramírez etal., 2000; Dominínguez-Bocanegra and Torres-Muñoz, 2004; Kim etal., 2007; Baeza et al., 2009). This yeast has also been mutated using UV radiation (Girard et al., 1997), ionising radiation (Sun et al., 2004), ethyl methanesulfonate (Calo et al., 1995; Flenø et al., 1997), methyl methanesulfonate (Bon et al., 1997) and benomyl (Calo et al., 1995).

Screening of the obtained mutants entails the visual appraisal of colour in colonies grown in a solid medium and the isolation of strains forming dark pink to claretcoloured colonies, i.e. potentially with a high concentration of astaxanthin. Next batch cultures of isolates are then run and the level of astaxanthin synthesis is evaluated. Due to the high frequency of reversion, the level of pigment synthesis should be monitored in several consecutive passages.

* Corresponding author: B. Stachowiak, Institute of Technology of Plant Origin Food, Poznan University of Life Sciences, Poland, ul. Wojska Polskiego 31, 60-624 Poznań, Poland; e-mail: bstach@up.poznan.pl 
Literature sources worldwide hardly ever report examples of $X$. dendrorhous mutants characterized by a spectacular concentration of astaxanthin in cells. It is rather believed that through mutagenesis it would be possible to obtain mutants with pigment concentrations of approx. two times higher in comparison to wild strains (Kim et al., 2007). A further increase in its efficiency is possible when applying an appropriately selected culture method. The tremendous effect of culture conditions on the production of astaxanthin is probably the reason why in recent years, literature sources worldwide have presented only testing results concerning the effect of medium composition and other environmental factors on the synthesis of this pigment by $X$. dendrorhous mutants. Detailed descriptions of mutations, as well as laborious selection procedures are frequently omitted or given in a very brief form (Bon et al., 1997; Ramírez et al., 2000; DominínguezBocanegra and Torres-Muñoz, 2004; Kim et al., 2007). A lack of exchange of expertise in this respect constitutes a considerable obstacle for all researchers conducting studies on obtaining highly-pigmenting strains of $X$. dendrorhous through mutagenesis.

In this paper mutations of the Xanthophyllomyces dendrorhous DSM 5626 strain were performed using mutagens, whose effect on the growth and carotenogenesis in this yeast has been relatively poorly documented in scientific literature on the subject. These mutagens include UV radiation, as well as benomyl, ethyl methanesulfonate and ethidium bromide. The aim of this study was to evaluate their effectiveness in generating astaxanthin-hyperproducing strains.

\section{Experimental}

\section{Materials and Methods}

Microorganism and culture conditions. Yeast Xanthophyllomyces dendrorhous DSM 5626 (= Phaffia rhodozyma ATCC 24202 and CBS 5905) from Deutche Sammlung von Mikroorganismen and Zellkulturen was employed as the parental wild-type strain in this study. It was maintained on YM slants ( $1 \%$ glucose, $0.5 \%$ peptone, $0.3 \%$ malt extract, $0.3 \%$ yeast extract and $2 \%$ agar for solid; $\mathrm{pH} 5.0$ ) at $4{ }^{\circ} \mathrm{C}$. Yeast grown on agar slants were flushed with $10 \mathrm{ml} \mathrm{YM}$ medium and the obtained suspension was used to inoculate fresh YM medium. Parental liquid cultures were grown for 4 days at $22^{\circ} \mathrm{C}$ in $250-\mathrm{ml}$ Erlenmeyer flasks (working capacity of $80 \mathrm{ml}$, including $5 \%$ inoculum) with continuous shaking $(250 \mathrm{rpm})$ under constant illumination of $600 \mathrm{~lx}$. Mutations were performed on the 4-day yeast suspension in the $\mathrm{YM}$ medium at $\mathrm{OD}=0.3-0.4$ $(\lambda=600 \mathrm{~nm})$.
UV mutagenesis. The volume of $100 \mu \mathrm{l}$ parental strain suspension was uniformly distributed over the surface of solidified YM medium (Petri dishes). Obtained samples were UV irradiated $(\lambda=254 \mathrm{~nm})$ for $0.5,1,1.5,2,2.5,5,10,15,30,45$ and 60 min. During sample exposure to UV radiation dishes, remained open and the distance from dishes to the source of radiation was $20 \mathrm{~cm}$. The control was comprised of samples not exposed to UV radiation.

Benomyl mutagenesis. The fungicide benomyl (metyl 1-(butylcarbamoyl)-2-benzimidazolecarbamate) was dissolved in DMSO and introduced into liquid YM medium with agar at $0.01,0.015,0.02,0.025,0.05$, $0.075,0.1,0.25,0.5,0.75,1,2.5,5,7.5,10,25,50$ and $100 \mathrm{mg} / \mathrm{ml}$ final concentration. The volume of $100 \mu \mathrm{l}$ of parental yeast suspension and $15 \mathrm{ml}$ of the medium with benomyl were poured into a Petri dish, mixed thoroughly and left until set. In control cultures an appropriate amount of DMSO was introduced to the medium.

Ethidium bromide mutagenesis. The volume of $100 \mu \mathrm{l}$ of parental yeast suspension and $15 \mathrm{ml}$ of liquid YM medium with agar were poured into a Petri dish and mixed thoroughly. After setting, wells of $10 \mathrm{~mm}$ in diameter were cut in the inoculated medium, to which $100 \mu \mathrm{l}$ aqueous ethidium bromide solution were poured at a $0.01,0.1,0.25,0.5,1,2.5,5,10$ and $25 \mathrm{mg} / \mathrm{ml}$ concentration. Distilled water was poured to the wells in control cultures.

Ethyl methanesulfonate mutagenesis. The volume of $5 \mathrm{ml}$ parental strain suspension was centrifuged and the precipitate was suspended in $1 \mathrm{ml}$ phosphate buffer ( $\mathrm{pH}$ 7.0). Samples were supplemented with ethyl methanesulfonate at $2 \%, 4 \%, 6 \%, 8 \%$ and $10 \%(\mathrm{v} / \mathrm{v})$ final concentration. Samples were incubated dynamically $(150 \mathrm{rpm})$ at room temperature for 15, 30, 60, 75 and $90 \mathrm{~min}$. After incubation physiological saline $(8 \mathrm{ml})$ was added to all samples in order to inhibit the effect of the mutagen on yeast cells. Cells were washed twice with physiological saline and the washed yeast biomass was suspended in $5 \mathrm{ml} \mathrm{YM}$ medium. The obtained yeast suspension was incubated for $24 \mathrm{~h}$ at $22^{\circ} \mathrm{C}$ and next inoculated on the solidified YM medium $(100 \mu \mathrm{l})$. The control comprised samples not subjected to the action of the mutagen.

Selection procedure. After the completion of the mutation plate cultures were incubated for 14 days at $22^{\circ} \mathrm{C}$ at a constant illumination of $600 \mathrm{~lx}$. Next colour and colouring intensity of grown colonies were evaluated visually. Strains with intense pink, raspberry and claret-coloured colonies were transferred onto agar slants. After 10 days batch cultures were run in order to evaluate the yeast growth and amount of produced carotenoids, including astaxanthin. Strains hyperproducing the pigment in comparison to the parental strain were passaged onto YM slants at 4-week intervals. Batch 
cultures were run for each passage. Mutant batch cultures were run for 5 days under identical conditions as those applied for the parental strain.

Carotenoid extraction and analysis. Pigments from yeast cells were isolated based on the method described by Sedmak et al. (1990), as modified by the author of this study. The volume of $10 \mathrm{ml}$ of culture was centrifuged and then the pellet was washed twice with distilled water and resuspended in $5 \mathrm{ml}$ of DMSO (preheated to $55^{\circ} \mathrm{C}$ ). The entire volume was vortexed for $30 \mathrm{~s}$ and next $5 \mathrm{ml}$ of the hexane fraction from petroleum were added. The volume was again vortexed for $30 \mathrm{~s}$ and $20 \% \mathrm{NaCl}$ aqueous solution was added in batches at $0.5 \mathrm{ml}$. The hexane phase with pigments was separated by centrifugation.

The total carotenoid and astaxanthin yields in samples were performed on a Waters Alliance HPLC System 2695. Analysis conditions of pigments were described by Gramza-Michałowska and Stachowiak (2010). Biomass was measured by DCW (dry cell weight).

Statistical analysis. The presented data were analysed by one-way analysis of variance (ANOVA). Differences between treatments were examined for the level of significance by Tukey's range test.

\section{Results}

UV radiation mutagenesis. Mutagenesis using UV radiation involved irradiation of the parental strain cells on solid YM medium. The cell exposure to UV radiation for a period shorter than $5 \mathrm{~min}$. did not result in a change in colour of grown yeast colonies in comparison to the control, although it affected their counts. As early as after 2 minutes the size of the yeast population was reduced by $50 \%$. Starting from the $5^{\text {th }}$ minute in plate cultures growth was observed only for single colonies, markedly changed phenotypically: claret, raspberry, pale pink, orange, white.

All pink, raspberry and claret-coloured strains (a total of approx. 50 strains) were isolated from plate cultures exposed to UV radiation for $5 \mathrm{~min}$ and longer. Growth of only eight of them was obtained on slants. One denoted as $26 \mathrm{UV}$, was capable of the synthesis of astaxanthin at a level significantly higher than the parental strain (Table I).

Benomyl mutagenesis. Mutations produced with the application of benomyl were obtained by introducing the mutagen to the growth medium. No growth of yeasts was observed on media containing $0.25-100 \mathrm{mg} / \mathrm{ml}$ of this compound. Single yeast colonies with different intensities of the pink colour grew on media containing 0.015 to $0.1 \mathrm{mg} / \mathrm{ml}$ benomyl.

Benomyl turned out to be effective in generating hyperpigmenting mutants. Among the 20 isolated strains a total of 14 produced carotenoids at a significantly higher level than the parental strain. Eight strains produced astaxanthin. A significantly higher pigment content in dry matter was recorded in five strains, i.e. 11B, 13B, 34B, 35B and 39B (Table I).

Ethidium bromide mutagenesis. Mutations generated with the use of ethidium bromide were provided by applying the well diffusion method. After 14 days of culture in the presence of the mutagen small, distinct lightened zones (no yeast growth) of max. $6 \mathrm{~mm}$ in size as well as adjacent zones of yeast growth inhibition (4-6 mm) were observed in samples, in which ethidium bromide was introduced to wells at a concentration of $0.5-2.5 \%$. In the case of the lower mutagen concentrations only yeast growth inhibition was observed $(5-15 \mathrm{~mm})$. In zones of yeast growth inhibition small colonies were detected, with colours distinctly different from the colour of parental strain colonies, i.e. claret, pinkish red, pink, as well as bigger, orange colonies.

Table I

Growth and carotenoid content in liquid cultures of parental strain Xanthophyllomyces dendrorhous DSM 5626 and its mutants generated as a result of mutations

\begin{tabular}{|c|c|c|c|c|c|c|c|c|c|}
\hline Analysed parameter & DSM 5626 & $26 \mathrm{UV}$ & $11 \mathrm{~B}$ & $13 \mathrm{~B}$ & $34 \mathrm{~B}$ & $35 \mathrm{~B}$ & $39 \mathrm{~B}$ & $9 \mathrm{BE}$ & 10BE \\
\hline Yeast dry matter $(\mathrm{g} / \mathrm{l})$ & $4.95^{\mathrm{c}}$ & $3.60^{\mathrm{a}}$ & $4.40^{\mathrm{b}}$ & $3.80^{\mathrm{a}}$ & $4.43^{\mathrm{b}}$ & $3.80^{\mathrm{a}}$ & $3.60^{\mathrm{a}}$ & $4.23^{\mathrm{b}}$ & $3.57^{\mathrm{a}}$ \\
\hline \multirow{3}{*}{$\begin{array}{l}\text { Carotenoids }(\mathrm{mg} / \mathrm{l}) \text { : } \\
\text { - total } \\
\text { - astaxanthin } \\
\text { - others }\end{array}$} & $1.03^{\mathrm{a}}$ & $1.36^{\mathrm{b}}$ & $1.48^{\mathrm{c}}$ & $1.57^{\mathrm{cd}}$ & $1.62^{\mathrm{d}}$ & $1.13^{\mathrm{a}}$ & $1.12^{\mathrm{a}}$ & $1.65^{\mathrm{d}}$ & $1.34^{\mathrm{b}}$ \\
\hline & $0.93^{\mathrm{a}}$ & $1.29^{\mathrm{b}}$ & $1.44^{\mathrm{c}}$ & $1.49^{\mathrm{cd}}$ & $1.51^{\mathrm{cd}}$ & $1.06^{\mathrm{a}}$ & $1.04^{\mathrm{a}}$ & $1.58^{\mathrm{e}}$ & $1.26^{\mathrm{b}}$ \\
\hline & 0.08 & 0.07 & 0.04 & 0.08 & 0.11 & 0.07 & 0.08 & 0.05 & 0.08 \\
\hline \multirow{3}{*}{$\begin{array}{l}\text { Carotenoids }(\mathrm{g} / \mathrm{kg}): \\
\text { - total } \\
\text { - astaxanthin } \\
\text { - others }\end{array}$} & $0.21^{\mathrm{a}}$ & $0.38^{\mathrm{d}}$ & $0.34^{\mathrm{c}}$ & $0.41^{\mathrm{e}}$ & $0.37^{\mathrm{d}}$ & $0.30^{\mathrm{b}}$ & $0.31^{b c}$ & $0.38^{\mathrm{d}}$ & $0.37^{\mathrm{d}}$ \\
\hline & $0.19^{a}$ & $0.36^{\mathrm{de}}$ & $0.33^{\mathrm{b}}$ & $0.39^{\mathrm{f}}$ & $0.34^{\mathrm{cd}}$ & $0.28^{\mathrm{b}}$ & $0.29^{\mathrm{b}}$ & $0.37^{\mathrm{ef}}$ & $0.35^{\text {cde }}$ \\
\hline & 0.02 & 0.02 & 0.01 & 0.02 & 0.03 & 0.02 & 0.02 & 0.01 & 0.02 \\
\hline Astaxanthin (\%) & $92^{\mathrm{a}}$ & $95^{\mathrm{bc}}$ & $97^{\mathrm{d}}$ & $95^{\mathrm{bc}}$ & $93^{\mathrm{ab}}$ & $94^{\mathrm{ab}}$ & $93^{\mathrm{ab}}$ & $97^{\mathrm{d}}$ & $94^{\mathrm{ab}}$ \\
\hline
\end{tabular}

Results in the table are means from two independent series of experiments (6 replications)

$\mathrm{a}, \mathrm{b}-$ represent significant differences at $\mathrm{P}<0.05$ for comparison in each row; $\mathrm{a}-$ the lowest value 
From those locations numerous strains were isolated, among which only 12 grew on agar slants. Of these strains four produced astaxanthin, although only two did so at a level significantly higher than the parental strain: 9BE and 10BE (Table I). A lack of growth on slants was observed particularly in case of dark claret colonies with diameters of max. $1 \mathrm{~mm}$.

Ethyl methanesulfonate mutagenesis. In the case of mutations generated by ethyl methanesulfonate biomass of parental yeasts was suspended in a solution containing the mutagen and after incubation yeasts were transferred onto YM medium containing no mutagen.

Only the parental strain cells in a solution of $6 \%$ ethyl methanesulfonate and higher resulted in a change of colour in the growing yeast colonies.

The lowest applied ethyl methanesulfonate concentration, i.e. $2 \%$, had no effect on yeast viability, irrespective of the time of yeast incubation in the mutagen solution. A reduction of viability by $50 \%$ in comparison to the control was recorded in the case of 45-minute incubation of parental yeast in a $4 \%$ mutagen solution. Due to a lack of changes in the appearance of colonies in comparison to the control, no strains were isolated for further experiments from plate cultures of yeasts incubated in $2 \%$ and $4 \%$ ethyl methanesulfonate solutions.

In the case of a $6 \%$ concentration the first changes in the appearance of colonies were observed in yeast cultures subjected to a 15-minute action of the mutagen. They were very small, less than $1 \mathrm{~mm}$ in diameter (vs. the diameter of parental strain colonies of 1-2 mm) and an intense pink and claret in colour. Unfortunately, attempts to isolate strains from those cultures were not successful, as isolates did not grow on agar slants. As a result of 30-minute suspension in a 6\% ethyl methanesulfonate solution viability of yeasts was by $50 \%$ lower in comparison to the control and the colonies were $2-3 \mathrm{~mm}$ in diameter. Growth of individual colonies was observed in cultures of yeasts exposed to the action of ethyl methanesulfonate for 45 and 60 minutes. There was no growth of colonies in cultures of yeasts kept for 75 and 90 minutes in a 6\% ethyl methanesulfonate.

After incubation of parental strain in $8 \%$ and $10 \%$ ethyl methanesulfonate solutions, no yeast growth was detected in plate cultures incubated with the mutagen for $45 \mathrm{~min}$ and longer. Growth of individual colonies (1-6 colonies), pink and claret in colour, was observed in the case of a shorter incubation time. An attempt at their isolation was also unsuccessful.

\section{Discussion}

In this study the isolation of over 500 mutants, salmon pink to claret in colour, was attempted. Some of them showed no growth on slants. Some mutants were lost during passaging as a result of a lack of growth in successive passages, loss of their ability to synthesise astaxanthin or carotenoids in general.

The problems with isolation and passaging of a considerable proportion of highly pigmented mutants may be associated with an aneuploid state. Some findings suggested that Xanthophyllomyces dendrorhous DSM 5626 is polyploid (Hermosilla et al., 2003) so lethal mutations would have to be induced at each copy of a gene for a given locus. It is much less probable than in the case of haploid. It can be supposed that mutagens used in the study could have induced an aneuploid state. Abnormalities in chromosome number usually lead to severe disorders in the functioning of cells, which often decrease the rate of cell proliferation and end in their death (Sheltzer and Amon, 2011).

But research indicates that the lethality of highly pigmented mutants $X$. dendrorhous may also be associated with reactive oxygen species (ROS) hyperproduction. It was suggested that mutations may occur in the mitochondrial genome or the nuclear genome coding mitochondrial proteins (Sun et al., 2004; Liu and Wu, 2006; Pfeiffer et al., 2010) which may cause disturbances in the mitochondrial function. Mitochondria are sources of ROS, which may affect gene expression in the carotenoid synthesis pathway (Potargowicz et al., 2005; Malik and Czajka, 2012). It was shown, that astaxanthin protects cells against oxidative stress improving mitochondrial function through retaining mitochondria in the reduced state. But under conditions of acute oxidative stress when large amounts of ROS are produced, astaxanthin showed no effect (Wolf et al., 2010). Thus excess ROS may cause sublethal damage of DNA or genes of the repair system (Dizdaroglu, 2012; Malik and Czajka, 2012). This may lead to the reduction of cell viability in the successive generations, and consequently to cell death.

Jointly on slants growth was observed for 40 carotenoid-producing strains, among which sixteen produced astaxanthin. In eight of them pigment content in dry matter was significantly higher in comparison with the parental strain and it did not change during the selection procedure (four successive passages). These strains were considered stable (Table I). Maximum astaxanthin concentration of $0.39 \mathrm{~g} / \mathrm{kg}$ was obtained for strain 13B. Although it is a value by $100 \%$ higher in comparison with the parental strain, it is still very low and may be considered doubtful when compared with literature data. However, it needs to be stressed here that in this study HPLC was applied for quantitative determinations of carotenoids and astaxanthin, while in scientific literature these analyses are mainly performed by spectrophotometry based on the value of molar absorptivity of 1600, established by Johnson et al. (1979) for $1 \%(\mathrm{w} / \mathrm{v})$ solution of astaxanthin in acetone in a $1-\mathrm{cm}$ 
cuvette. Some researchers also apply the value of 2100 , which is obtained by measuring the astaxanthin standard by Hoffman-La Roche. According to measurements taken within this study the value of molar absorptivity for $1 \%$ astaxanthin solution in hexane of the petroleum fraction is 3100 . Thus when comparing results reported in literature sources with those found in this study we need to multiply the latter by the correction for the difference in the extinction coefficient, i.e. $3100 / 1600$ (1.94) or 3100/2100 (1.5).

The problem of low efficiencies of carotenoid and astaxanthin determinations, connected with the adopted assay method, has been repeatedly discussed in scientific literature on the subject. For example Flenø et al. (1999), using HPLC for quantitative determinations of carotenoids produced by P. rhodozyma CBS 5905, i.e. the parental strain investigated in this study, obtained low astaxanthin efficiencies, similar to those recorded in this study. Similarly, Vazquez et al. (1997) in the same strain culture on YM, under almost identical conditions as those adopted in this study, recorded a low concentration of astaxanthin in dry substance, amounting to $0.20 \mathrm{~g} / \mathrm{kg}$. They also used HPLC in quantitative determinations of carotenoids. Authors of those studies proposed a clear suggestion that the application of a spectrophotometric method in quantitative analyses of pigments leads to an overestimation of their efficiencies. When using this method Fontana et al. (1996), Yamane et al. (1997) and Chumpolkulwo et al. (1997) obtained astaxanthin efficiency of $0.3-0.5 \mathrm{~g} / \mathrm{kg}$ for $X$. dendrorhous DSM 5626.

Among the tested mutagens benomyl turned out to be most effective in generating astaxanthin-overproducing mutants. Mutations obtained with the use of this mutagen generated five strains, which produced astaxanthin at $0.28-0.39 \mathrm{~g} / \mathrm{kg}$ in dry matter, i.e. $50-100 \%$ more than the parental strain. Mutations of the yeast $X$. dendrorhous with the use of benomyl were also carried out by Calo et al. (1995). As a result they generated strain B10 producing only by $30 \%$ more astaxanthin $(0.37 \mathrm{~g} / \mathrm{kg}$ as determined by HPLC) than the parental strain, although the total amount of pigments produced by that strain increased two-fold.

In the experiments conducted within this study a very high sensitivity towards benomyl was found in the parental strain. The experimental value for $\mathrm{ED}_{50}$ was $0.01 \mathrm{mg} / \mathrm{ml}$. An identical sensitivity of X. dendrorhous to benomyl was also reported in their experiments by Calo et al. (1995).

The application of ethyl methanesulfonate to provide mutations of $X$. dendrorhous DSM 5626 in this study was unsuccessful due to a lack of growth of potentially highly pigmenting isolates on agar slants. More effective isolations of mutants obtained as a result of ethyl methanesulfonate treatment were performed within a study by the above mentioned Calo et al. (1995). However, isolates obtained by those researchers proved to be hypoproducers of astaxanhin. They produced 25-70\% less pigment when compared to the parental strain.

In the case of UV radiation in this study one stable strain 26UV was generated, producing astaxanthin at a significantly higher level than the parental strain. Girard et al. (1997) were of an opinion that UV light is effective in generating strains synthesising only $\beta$-carotene. The above mentioned authors consistently stressed very low survival rates of the yeast $X$. dendrorhous during exposure to UV radiation. Girard et al. (1997) recorded a population size of the tested yeast $X$. dendrorhous reduced by $90 \%$ as early as after $50 \mathrm{~s}$ irradiation. In this study such an effect was observed after $180 \mathrm{~s}$.

As a result of mutations produced using ethidium bromide a considerable number of small dark pink and claret-coloured colonies were observed on the medium with an addition of the mutagen. They were very difficult to isolate and as a result only two stable strains were obtained, overproducing astaxanthin in comparison with the parental strain, i.e. $9 \mathrm{BE}$ and 10BE. As it was reported by Pfeiffer et al. (2010), ethidium bromide inhibits the synthesis of mitochondrial DNA (mtDNA) molecules, as well as stimulates the decomposition of already existing mtDNA molecules. As a result respiration deficient mutants were produced. Pfeiffer et al. (2010) observed such respiration deficient mutants of P. rhodozyma exposing strain CBS 5905 to ethidium bromide. They grew in a manner identical to that within this study in the form of small claret-coloured colonies. Those authors claimed that mutations occurred in the mitochondrial genome.

Conclusions. Astaxanthin-overproducing strains of Xanthophyllomyces dendrorhous are a promising perspective for industry. Overproduction of this pigment can be obtained by wild yeast mutagenesis. However not all mutagens are effective for this purpose. In the present study, ethyl methanesulfonate was completely useless. Another problem is the difficult or impossible isolation of astaxanthin-overproducing strains of Xanthophyllomyces dendrorhous, as well as too low survival during passage.

In this study eight stable mutants were generated, including five with benomyl. They exhibited increases up to $100 \%$ in astaxanthin contents in comparison to the parental strain. Astaxanthin production efficiencies can be further improved through optimization of culture conditions for the produced mutants.

\section{Acknowledgements}

The author is grateful to the Ministry of Science and Higher Education, Poland (grant no 2 PO6T 024 30) for the financial support of this work. Many thanks to Dr Maciej Wnuk (The University of Rzeszów, Poland) for helpful discussion. 


\section{Literature}

Baeza M., P. Retamales, D. Sepúlveda, P. Lodato, A. Jiménez and V. Cifuentes. 2009. Isolation, characterization and long term preservation of mutant strains of Xanthophyllomyces dendrorhous. J. Basic. Microbiol. 49: 135-141.

Bon J.A., T.D. Leather and R.K Jayaswal. 1997. Isolation of astaxanthin-overproducing mutants of Phaffia rhodozyma. Biotechnol. Lett. 19: 109-112.

Breithaupt D.E. 2007. Modern application of xanthophylls in animal feeding - a review. Trends in Food Sci. Technol. 18: 501-506.

Calo P., J.B. Velázquez, C. Sieiro, P. Blanco, E. Longo and T.G. Villa. 1995. Analysis of astaxanthin and other carotenoids from several Phaffia rhodozyma mutants. J. Agric. Food. Chem. 43: 1396-1399.

Chumpolkulwong N., T. Kakizono, S. Naga and N. Nishio. 997. Increased astaxanthin production by Phaffia rhodozyma mutants isolated as resistant to difenylamine. J. Ferment. Bioeng. 83: 429-434.

Dizdaroglu M. 2012. Oxidatively induced DNA damage: Mechanisms, repair and disease. Cancer. Lett. 327: 26-47.

Dominíguez-Bocanegra A.R and J.A. Torres-Muñoz. 2004. Astaxanthin hyperproduction by Phaffia rhodozyma (now Xanthophyllomyces dendrorous) with raw milk as sole source of energy. Appl. Microbiol. Biotechnol. 66: 249-252.

Flenø B., I. Christensen, R. Larsen, S.R. Johansen and E. Johnson. 1997. Astaxanthin-producing yeast cells, methods for their preparation and their use. US Patent No. 5, 599, 711.

Fontana J.D., M.F. Guimrães, N.T. Martins, C.A. Fontana and M. Baron. 1996. Culture of the astaxanthinogenic yeast Phaffia rhodozyma in low-cost media. Appl. Biochem. Biotechnol. 57: 413-422. Girard P., C.E.J. Javelot and B.D.V. Vladescu. 1997. Phaffia rhodozyma mutants, process for producing $\beta$-carotene and use of $\beta$-carotene rich biomass. US Patent No. 5, 691,190.

Golubev W.I. 1995. Perfect state of Rhodomyces dendrorhous (Phaffia rhodozyma). Yeast 11: 101-110.

Goswami G., S. Chaudhuri and D. Dutta. 2010. The present perspective of astaxanthin with reference to biosynthesis and pharmacological importance. World. J. Microbiol. Biotechnol. 26: 1925-1939. Gramza-Michałowska A. and B. Stachowiak. 2010. The antioxidant potential of carotenoid extract from Phaffia rhodozyma. Acta Sci. Pol. Technol. Aliment. 9: 171-188.

Hermosilla G., C. Martínez, P. Retamales, R. León and V. Cifuentes. 2003. Genetic determination of ploidy level in Xanthophyllomyces dendrorhous. Antonie van Leeuwenhoek 84: 279-287.
Johnson E.A. and M. Lewis. 1979. Astaxanthin formation by the yeast Phaffia rhodozyma. J. Gen. Microbiol. 115: 173-183.

Kim S.K., J.H. Lee, C.H. Lee and Y.C. Yoon. 2007. Increased carotenoid production in Xanthophyllomyces dendrorhous G276 using plant extracts. J. Microbiol. 45: 128-132.

Liu Y.S. and J.Y. Wu. 2006. Hydrogen peroxide-induced astaxanthin biosynthesis and catalase activity in Xanthophyllomyces dendrorhous. Appl. Microbiol. Biotechnol. 73: 663-668.

Malik A. and A. Czajka. 2012. Is mitochondrial DNA content a potential biomarker of mitochondrial dysfunction? Mitochondrion DOI: MITOCH-00764.

Pfeiffer I., C. Vágvölgy, T. Hirano and J. Kucsera. 2010. Characterization of petite mutants of the basidiomycetes Phaffia rhodozyma CBS 5905. Acta Biol. Szeged. 54: 143-148.

Potargowicz E., E. Szerszenowicz, M. Staniszewska and D. Nowak. 2005. Mitochondria as a source of reactive oxygen species. Postepy Hig. Med. Dosw. 59: 259-266.

Ramírez J., M.L. Nuñez and R. Valdivia. 2000. Increased astaxanthin production by a Phaffia rhodozyma mutant grown on date juice from Yucca fillitera. J. Ind. Microbiol. Biotechnol. 24: 187-190.

Rodríguez-Sáiz M. and J.L. de la Fuente. 2010. Xanthophyllomyces dendrorhous for the industrial production of astaxanthin. Appl. Microbiol. Biotechnol. 88: 645-658.

Sedmak J.J., D.K. Weerasinghe and S.O. Jolly. 1990. Extraction and quantification of astaxanthin from Phaffia rhodozyma. Biotechnol. Tech. 4: 107-112.

Sheltzer J.M., A. Amon. 2011. The aneuploidy paradox: costs and benefits of an incorrect karyotype. Trends. Genet. 27: 446-453.

Sun N., S. Lee and K.B. Song. 2004. Characterization of a carotenoid-hyperproducing yeast mutant isolated by low-dose gamma irradiation. Int. J. Food. Microbiol. 94: 263-267.

Vazquez M., V. Santos and J.C. Parajo. 1999. Effect of the source on carotenoid profiles of Phaffia rhodozyma strains. J. Indust. Microbiol. Biotechnol. 19: 263-268.

Wolf A.M., S. Sadamitsu, H. Hiranuma, I. Ohsawa, K. Iio, A. Satou, M. Ishikura and S. Ohta. 2010. Astaxanthin protects mitochondraial redox state and functional integrity against oxidative stress. J. Nutr. Biochem. 21: 381-389.

Yamane Y.I., K. Higashid, Y. Nakashimada, T. Kakizono and N. Nishio. 1997. Astaxanthin production by Phaffia rhodozyma enhanced in fed-batch culture with glucose and ethanol feeding. Biotech. Lett. 19: 1109-1111.

Yuan J.P., J. Peng, K. Yin and J.H. Wang. 2011. Potential healthpromoting effects of astaxanthin: A high-value carotenoid mostly from microalgae. Mol. Nutr. Food. Res. 55: 150-165. 